\title{
Aortic Impingement in Displaced Traumatic Spine Fracture with Complete Spinal Cord Transection: A Case Report
}

\author{
Asma Anan Mohammed ${ }^{a}$ Fatima R. Shulaiba ${ }^{a}$ Mina Hikmat Ismaeal Alhety ${ }^{a}$ \\ Hayder Saleh Abdul Hadi Al Saadi ${ }^{\text {b }}$ Bilal El Yafawi ${ }^{\text {b }}$ \\ aMedical Internship Program, Medical Education Department, Dubai Health Authority, Dubai, United Arab Emirates; \\ bTrauma/Orthopedics Department, Rashid Hospital, Dubai Health Authority, Dubai, United Arab Emirates
}

\section{Keywords}

Aortic impingement · Complete spinal transection · Spinal

cord trauma Thoracic spine fracture $\cdot$ Case report

\begin{abstract}
Aortic impingement associated with traumatic thoracic spinal fractures is a rare and potentially lethal complication that creates management challenges in an already complex clinical problem. Traumatic aortic injury is one of the leading causes of death in blunt trauma. Magerl divided thoracic and lumbar fractures into 3 categories; the primary focus of this report, type $C$ fractures, describes rotational injury and is one of the less common types, especially associated with aortic impingement as such. In this case, a young man was admitted following a near-fatal fall resulting in blunt force trauma to the midthoracic region. Emergency $C T$ revealed a type $C$ complete transection at the level of T11 and a grade I aortic injury. Definitive fixation of the spinal injury was delayed in favor of preventing further vascular injury by prioritizing the securing of hemodynamic stability. In traumatic thoracolumbar injuries, blunt traumatic aortic injury is often managed conservatively. However, blunt thoracic aortic injury is one of the leading causes of death from trauma, and each
\end{abstract}

karger@karger.com www.karger.com/dmj

Karger $\stackrel{\text { ' }}{5}$
(C) 2021 The Author(s)

Published by S. Karger AG, Basel

This is an Open Access article licensed under the Creative Common Attribution-NonCommercial-4.0 International License (CC BY-NC) (http://www.karger.com/Services/OpenAccessLicense), applicable to the online version of the article only. Usage and distribution for commercial purposes requires written permission. case requires its own case-by-case multidisciplinary management. In this occasion, management of the vascular insult was paramount to ensuring patient survival and favorable outcome.

(C) 2021 The Author(s).

Published by S. Karger AG, Basel

\section{Introduction}

Blunt thoracic aortic injury (BTAI) is a rare complication that may occur in association with traumatic spinal cord injuries, though the incidence is unknown. Generally, BTAI has been identified as a leading cause of death in blunt trauma [1]. Few cases have been reported in the literature. Additionally, few studies outline the appropriate management of aortic injury in unstable thoracic vertebral column fractures [2]. Various grading systems have been proposed with effort to guide therapy, and the widely accepted protocols will be presented in this study.

Traumatic spinal cord injuries are life-altering neuromechanical conditions that can have devastating longstanding physical, mental, and social effects. Spinal cord injury is a term encompassing injury of the spinal cord from the foramen magnum to the cauda equina which

Correspondence to:

Asma Anan Mohammed, asmamohd2@gmail.com 
occurs as a result of compulsion, incision, or contusion [3]. In this case report, we present an unstable, displaced, hyperextension thoracic spinal fracture with complete spinal cord transection and concomitant aortic impingement in a young patient.

\section{Case Description}

A young, previously healthy male in his mid-30s was brought to the emergency department after a midthoracic blunt force trauma caused by a fall from a height followed by impact with the edge of a railing. It was accompanied by a loss of consciousness, and the patient was found hypotensive at the scene by paramedics and resuscitated.

In the emergency department, the patient was tachycardic (112 bpm) and hypotensive $(87 / 41 \mathrm{~mm} \mathrm{Hg})$ with complaints of back pain and right-sided chest pain of 5/10 severity. Primary survey revealed bilaterally reduced air entry at the lung bases and tenderness on the right lower chest. Secondary survey exhibited a noticeable bulge at the mid-lower thoracic region with no external bleeding. Focused assessment with sonography for trauma was positive, and $1,700 \mathrm{~mL}$ of blood was drained after an intercostal drainage (ICD) tube was inserted on the right side. Neurological examination revealed paraplegia with sensation level at the umbilicus. Vascular examination showed delayed capillary refill and intact peripheral pulses (femoral and pedal pulses).

A polytrauma CT scan revealed complete transverse fracture dislocation of the T11 vertebra involving the vertebral body and posterior elements with total anterior and left displacement of the inferior fracture fragment and resultant complete transection of the spinal cord at that level. The anteriorly and left-displaced fragment was noted indenting the posterior aspect of the aorta without any visible contrast extravasation. Additionally, displaced fractures of the left transverse processes of $\mathrm{T} 9$ and $\mathrm{T} 10$ with a fracture of the spinous process of T12 were noted. The fractures of the T9 vertebra, T10 and T11 left transverse processes, and T12 spinous process indicate a thoracolumbar spine injury with a morphology classification of type $\mathrm{C}$ and a neurologic status of $\mathrm{N} 4$ according to the AOSpine Trauma Classification.

In the chest, bilateral hemopneumothorax was described with significant reduction of right lung volume, lower lobe collapse, left lower lobe laceration, and multiple displaced fractures in the left 9 th, 10th, and 11th ribs. There was also a periaortic hematoma with pneumomediastinum (Fig. 1, 2).

A provisional diagnosis of closed fracture dislocation of the thoracic spine was made. Due to the critical condition of the patient, a decision was made to delay operative treatment in favor of achieving hemodynamic stability. The patient was admitted to the surgical ICU under observation, sedated, intubated, and placed on mechanical ventilation with a high setting of inotropes. During this period, a further $1,400 \mathrm{~mL}$ of fluid was drained through the ICD tube. A CT angiography (CTA) revealed a grade I aortic injury (intimal tear) of the descending thoracic aorta at the level of T11 (Fig. 3). The intimal tear could not be measured. A multidisciplinary team meeting was held with trauma, vascular, thoracic surgery, and interventional radiology teams. A decision was made to opt for spinal fixation in prone positioning with conversion to supine if needed.

Aortic Impingement in Thoracic Spine

Fracture with Spinal Cord Transection

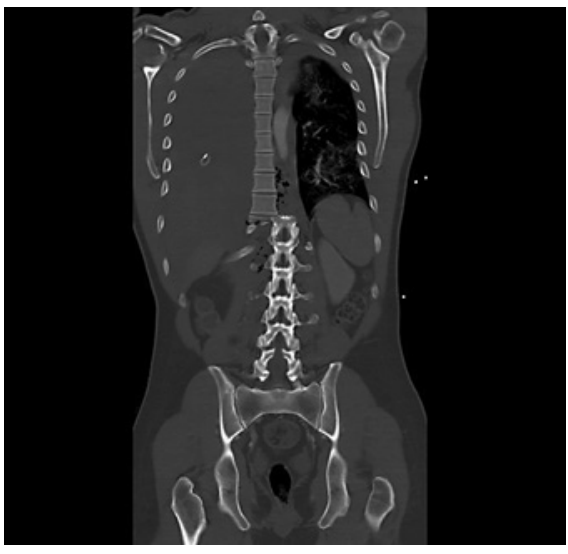

Fig. 1. Contrast-enhanced CT polytrauma, coronal plane, demonstrating a complete transverse fracture of the T11 vertebra with total displacement of the inferior fragment. Bilateral hemopneumothorax with markedly reduced right lung volume can also be observed.

The patient underwent surgical fixation with vascular, interventional radiology, and cardiothoracic teams on standby. In the case of T11-T12 fixation, the aim was to reduce and fixate the fracture without causing aortic injury, while simultaneously reducing the aorta back to its original position. Initially, the patient was placed in the prone position, without any traction or manipulation. A longitudinal posterior incision was made, running from T11 to L1, followed by dissection as per the procedure until the spinal column was reached and the fracture dislocation site was identified. During this, complete transection of the spinal cord was noted.

Four Schanz screws of $40 \times 6.2 \mathrm{~mm}$ were applied (USS 1 from Dupuy Sythes) in T9 and T10-2 pedicles of each vertebra. Four Schanz screws of $45 \times 6.2 \mathrm{~mm}$ were applied in T12 and L1-2 pedicles of each vertebra. All of the aforementioned screws were placed without reduction, after which the screws were used as joysticks to maneuver and reduce the fracture gradually over 20 min until acceptable reduction was achieved without compromising the hemodynamic status of the patient. The screws were then fixated with 1 metallic rod on each side, as well as a cross rod to ensure maximal stability. The wound was then closed in layers as per the procedure (Fig. 4-6).

There were no complications, and the patient was hemodynamically stable intra- and postoperatively. The patient regained normal capillary refill and continued to have intact peripheral pulses. Follow-up with CT aortogram 3 days postoperatively showed an intact contrast-opacified thoracic aorta with normal contour and no evidence of filling defects, dissection, or pseudoaneurysm. The metallic hardware artefacts are partially degrading the image quality. There is clear reduction of bony compression on the external aortic contour following the fixation of the spinal fracture (Fig. 7).

Following the surgery, a repeat CXR showed persistent mild to moderate pleural effusion on the left side, and a further $1,600 \mathrm{~mL}$ of blood was drained after an ICD tube was inserted on the left side. 
Fig. 2. Contrast-enhanced CT polytrauma, sagittal plane, bone view, demonstrating displaced transverse fracture of T11 (a) and thoracic aortic impingement (b) by the anteriorly and left-displaced inferior fracture fragment.

Fig. 3. Contrast-enhanced CT polytrauma with angiography sagittal plane demonstrating aortic impingement by the displaced distal fracture segment of T11 (a) and transverse plane demonstrating thoracic aortic impingement by the anteriorly and left-displaced inferior fracture fragment (b).
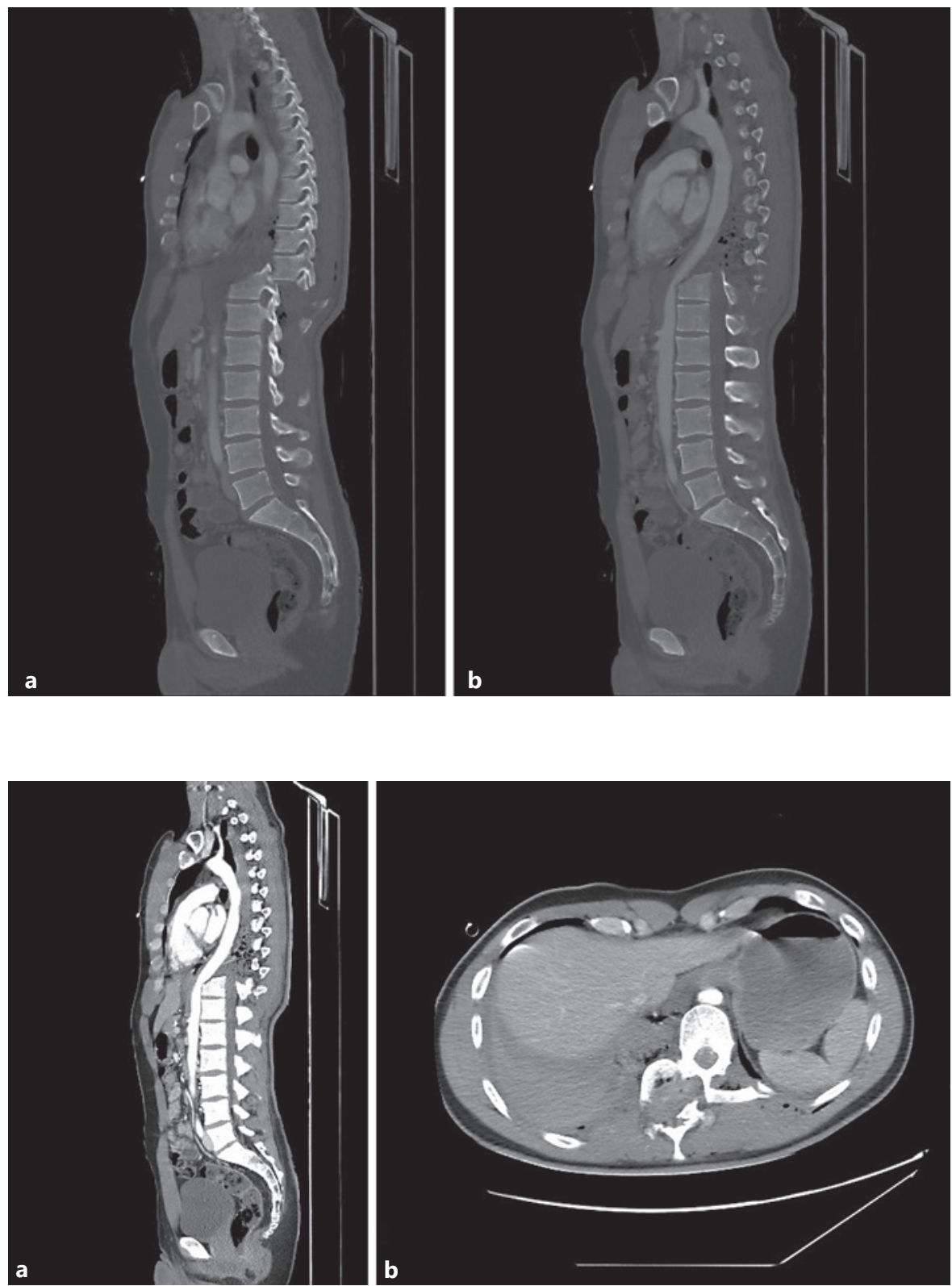

The patient was discharged in stable condition and will undergo rehabilitation therapy to improve function, prevent contractures, and achieve independence.

\section{Discussion}

Few cases of BTAI with traumatic spinal injuries have been reported in the literature such as that by Fattahi et al. [4], who described a hyperextension thoracic spine fracture complicated by paraplegia, aortic dissection, and right hemothorax requiring ICD tube insertion. Cultrera et al. [5] described a compression/distraction spinal fracture complicated by aortic encroachment without wall disruption. Bashir et al. [6] described an aortic laceration following a T10 vertebral body fracture. Murakami et al. [7] described a compression thoracic spine fracture complicated by aortic compression and pseudoaneurysm.

Thoracic and lumbar fractures of the spine were classified by Magerl et al. [8] into 3 types - A, B, and C-based on pathomorphology, each of which is divided into groups and subgroups. Type A injuries include vertebral 


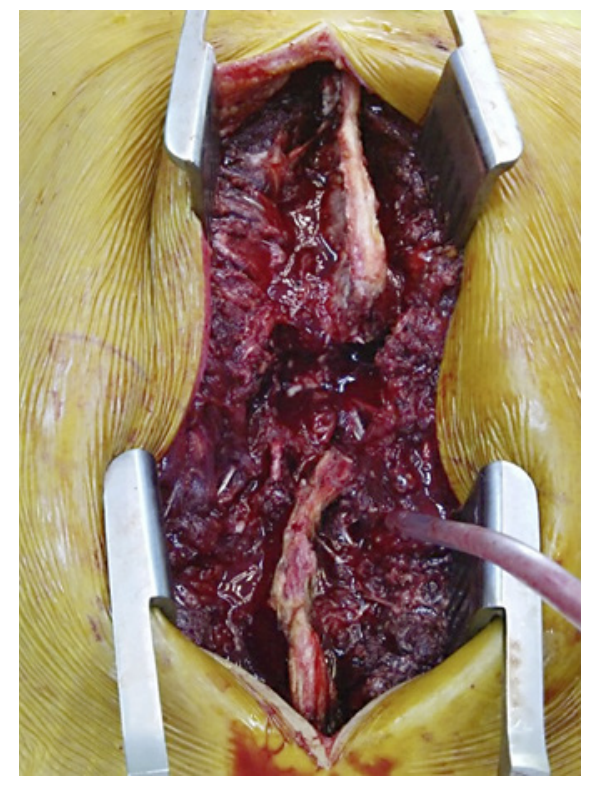

Fig. 4. Intraoperative image demonstrating vertical incision at the level of T9-L1 with skin retraction. The complete transection of the spinal cord is shown.

Fig. 6. Intraoperative fluoroscopy showing lateral view of the thoracolumbar spine with reduced fracture.

Fig. 7. Postoperative computed tomography aortogram, sagittal plane, showing an intact contrast-opacified thoracic aorta with normal contour and no evidence of filling defects, dissection, or pseudoaneurysm. The metallic hardware artefacts are partially degrading the image quality.
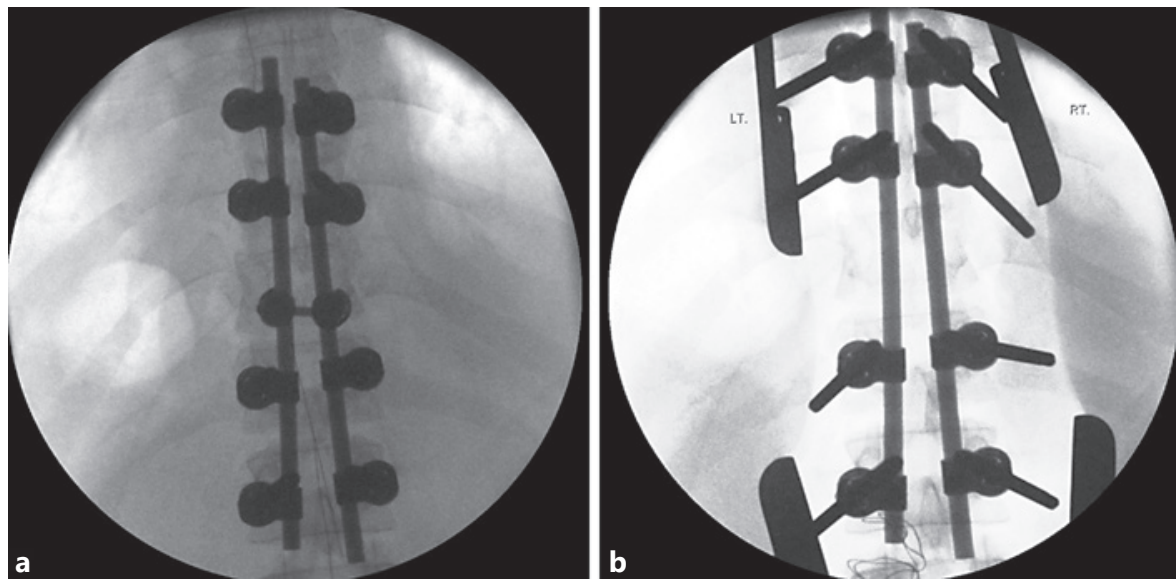

Fig. 5. a, b Intraoperative fluoroscopy showing AP view of the thoracolumbar spine showing reduction and fixation of the fracture done in accepted alignment.

6

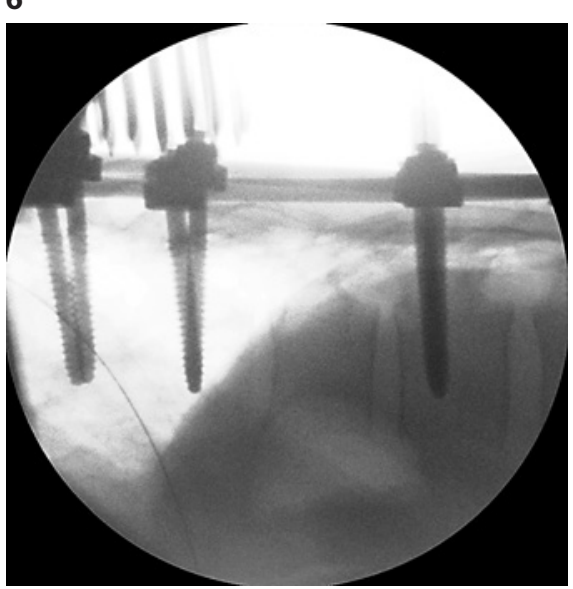

7

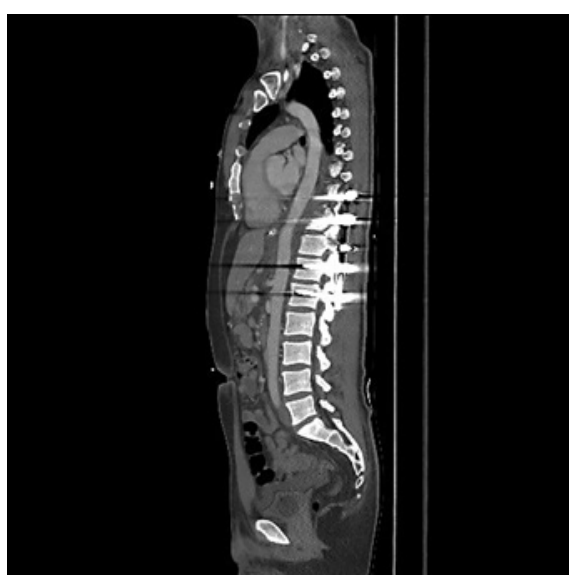

body injuries, type B lesions include disruption with anterior or posterior element involvement, and type $\mathrm{C}$ focuses on rotation with anterior or posterior element injuries. Type A fractures are the most common and make up $66 \%$ of all spinal injuries.

The current AOSpine Trauma Classification, which improves on the classification developed by Magerl et al. [8], divides the spinal column into 4 different segments: upper cervical spine $(\mathrm{C} 0-\mathrm{C} 2)$, subaxial cervical spine (C3-C7), thoracolumbar spine (T1-L5), and sacral spine (S1-S5 and the coccyx) to describe increasing levels of instability [9]. Three components are considered for every fracture, including morphology of the injury, neurologic status, and presence of clinical modifiers. Morphology of the injury may be divided into A (compression involving the anterior portion of the vertebral column), B (distraction involving the anterior and posterior tension band), and C (displacement or dislocation in any direction). Neurologic status is assessed at the initial examination and may be described as N0 (intact neurologic status), N1 (transient neurologic signs), N2 (signs of radiculopathy), N3 (incomplete spinal cord or cauda equina 
Table 1. The thoracolumbar AOSpine injury score

\begin{tabular}{ll}
\hline Classification & Points \\
\hline $\begin{array}{l}\text { Morphology of the injury } \\
\text { Type A - compression fractures }\end{array}$ \\
A0 \\
A1 & \\
A2 & 0 \\
A3 & 1 \\
A4 & 2 \\
Type B - distraction with tension band injuries & 3 \\
B1 & 5 \\
B2 & 5 \\
B3 & 6 \\
Type C-displacement/translational fractures & 7 \\
C & \\
Neurologic status & 8 \\
N0 & \\
N1 & 0 \\
N2 & 1 \\
N3 & 2 \\
N4 & 4 \\
Nx & 4 \\
Clinical modifiers & 3 \\
M1 & \\
M2 & \\
\hline
\end{tabular}

injury), $\mathrm{N} 4$ (complete spinal cord injury), or $\mathrm{Nx}$ (status cannot be assessed). The thoracolumbar AOSpine injury score (TL AOSIS) aims to award points to assign fracture severity and guide surgical treatment[10] (Table 1).

A patient with thoracic or lumbar fractures can present with severe back pain that worsens with movement. Some may experience motor or sensory dysfunction, as well as autonomic problems such as bowel or bladder dysfunction. Numbness, tingling, or weakness of the limbs can also occur.

The most sensitive and accurate modality for diagnosis of thoracolumbar injuries is the CT scan [11]. A supplemental MRI scan can be used to evaluate the surrounding soft tissues [12]. Spinal injuries require management by an interdisciplinary/multispecialty team composed of specialists in spine surgery, endovascular surgery, cardiothoracic surgery, interventional radiology, and anesthesia. Several treatment algorithms exist, with the latest guidelines formed according to the AOSpine thoracolumbar spine injury score. The algorithm recommends nonoperative care for fractures with a TL AOSIS score of 3 or less, operative or nonoperative treatment for injuries with a TL AOSIS of 4 or 5 , and operative treatment for fractures scoring $>5$ [10]. A multidisciplinary evaluation allows for preoperative patient selection and discussion on the need for prophylactic vascular interventions [13].

BTAI was first reported by Andreas Vesalius [14] in 1557 and is now a leading cause of death from trauma. Though multiple publications have since described BTAI in association with thoracic and lumbar spinal fractures, this presentation remains uncommon and carries a very high mortality rate if not managed appropriately. The mechanism of such concomitant aortic injuries remains unclear, although current theories suggest a combination of mechanical forces such as acute aortic stretching and hydraulic forces such as a sudden increase in intraluminal pressure [15]. Another theory suggests the transmission of distractive forces along the spinal anterior longitudinal ligament to the aorta [7]. A comprehensive literature review carried out by Santoro et al. [16] reported a mortality rate of $30 \%$, with distraction being more likely to cause intimal damage of the aorta while flexion-distraction injuries more commonly resulted in a pseudoaneurysm.

Vascular injury is diagnosed by CTA scan imaging, as the classic findings of widened mediastinum and hemopneumothorax on a screening CXR are not sensitive [17]. Inaba et al. [18] identified 3 types of aortic disruption hemodynamically unstable full thickness laceration, symptomatic intimal dissection with occlusion, and asymptomatic intimal dissection without occlusion. The anatomic classification of aortic injuries was defined by the Society of Vascular Surgery as follows:

- Ascending aorta: injuries proximal to the innominate artery.

- Aortic arch: injuries between the innominate artery and left subclavian artery.

- Isthmus: injuries arising $<1 \mathrm{~cm}$ of the ligamentum arteriosum.

- Descending thoracic aorta: injuries arising $>1 \mathrm{~cm}$ from the ligamentum arteriosum to the diaphragmatic hiatus.

- Abdominal aorta: injuries between the diaphragmatic hiatus and aortic bifurcation.

The Society of Vascular Surgery (SVS) proposed a classification system which classifies intimal tears as grade I, intramural hematomas as grade II, aortic pseudoaneurysm as grade III, and free aortic rupture as grade IV [19]. While this classification does not guide therapy, it offers recommendations for the management of aortic injuries. A conservative approach with expectant management and serial imaging is recommended for grade I injuries as most heal spontaneously, while thoracic endovascular aortic repair (TEVAR) is preferred for grade II-IV aortic injuries prior to spinal fixation in order to avoid uncontrolled intraoperative bleeding [20]. 
The Western Vascular Society (WVS) has since proposed a simplified grading of BTAI into minimal (SVS grade I and II injuries with intimal tear $<10 \mathrm{~mm}$ and no external contour abnormality), moderate (SVS grade III injuries with external contour abnormality or intimal tear $>10 \mathrm{~mm}$ ), and severe (SVS grade IV injuries with active extravasation or hematoma) [21]. The WVS recommends nonoperative management for minimal injuries, semielective repair of moderate injuries within $24-72 \mathrm{~h}$ with stabilization of concomitant injuries, and immediate repair of severe injuries.

TEVAR is preferred over open repair, provided anatomic suitability is established, as it carries lower risks of death and spinal cord ischemia [22]. In addition, the Eastern Association for the Surgery of Trauma (EAST) strongly recommends endovascular repair for patients who do not have any contraindications to the procedure [23], and the choice of treatment is guided by the hemodynamic condition of the patient, presence of other diseases, and previous surgical history.

A wide variety of complications can arise after endoscopic repair such as thromboembolism, spinal cord ischemia, device migration, device degradation, graft failure, deformation, and obstruction. The Society of Vascular Surgery (SVS) recommends systemic heparinization on an individualized basis after weighing the risks of bleeding versus thromboembolic complications [20]. When warranted, surgical repair of the graft may be done.

The follow-up protocol after endovascular repair includes imaging by CT scan at 1, 6, and 12 months and then annually subsequently. Injuries may resolve (return to normal aortic diameter with absence of external contour abnormality), remain stable (no interval change), or progress (enlargement in the size of injury). Minimal injuries are no longer imaged routinely due to high rates of spontaneous healing on follow-up. Some suggestions of the SVS include reduction of imaging frequency to every $2-5$ years provided no abnormalities are reported in the first 12-36 months, as well as replacing CTA with a combination of multiview chest X-ray and magnetic resonance angiography.

\section{Conclusion}

BTAI due to aortic impingement is a rare complication of traumatic thoracolumbar spinal fractures, with only a few cases reported in the existing literature, and poses a daunting medical challenge requiring a multidisciplinary team approach to achieve optimal results. As illustrated by our case, the hemodynamic stability and prevention of further vascular injury must take precedence over definitive fixation of the spinal fracture. Adequate investigation of the extent of vascular injury through CTA followed by endovascular repair, if indicated, is therefore imperative in the prevention of fatal vascular complications.

\section{Statement of Ethics}

All personally identifying information, such as name, was excluded from the study. Written informed consent was obtained from the patient for publication of this case report and any accompanying images.

\section{Conflict of Interest Statement}

The authors have no conflicts of interest to declare.

\section{Funding Sources}

There are no funding sources or sponsors for this report.

\section{Author Contributions}

A.A.M. contributed to writing - original draft, review and editing, and manuscript preparation. F.R.S. contributed to writing original draft, review and editing, and selection of references. M.H.I.A. contributed to writing - original draft, review and editing, and selection of references. H.S.A.H. contributed to supervision, project administration, and coordination. B.E.Y. contributed to supervision.

\section{Data Availability Statement}

All data generated or analyzed during this study are included in this article. Further enquiries can be directed to the corresponding author.

\section{References}

1 Arthurs ZM, Starnes BW, Sohn VY, Singh N, Martin MJ, Andersen CA. Functional and survival outcomes in traumatic blunt thoracic aortic injuries: an analysis of the National Trauma Databank. J Vasc Surg. 2009;49(4): 988-94.

2 Chock MM, Aho J, Naik N, Clarke M, Heller $S$, Oderich GS. Endovascular treatment of distal thoracic aortic transection associated with severe thoracolumbar spinal fracture. Vascular. 2015;23(5):550-2. 
3 Nas K, Yazmalar L, Şah V, Aydın A, Öneş K. Rehabilitation of spinal cord injuries. World J Orthop. 2015;6(1):8-16.

4 Fattahi A, Mohajeri SMR, Daneshi A, Shahivand A. Hyperextension thoracic spine fracture with complete neurological recovery after surgical fixation: a case report. Surg Neurol Int. 2020;11:137.

5 Cultrera F, Gamberini E, Iacono G, Turicchia GU, Agnoletti V, Tosatto L. Unstable thoracic spine fracture with aortic encroachment: a potentially fatal association and a suggested treatment. Int J Surg Case Rep. 2017;39:1814.

6 Bashir M, McWilliams RG, Desmond M, Kuduvalli M, Oo A, Field M. Blunt aortic injury secondary to fragmented tenth thoracic vertebral body. Ann Thorac Surg. 2013;95(6): 2161-4.

7 Murakami R, Tajima H, Ichikawa K, Kobayashi Y, Sugizaki K, Yamamoto K, et al. Acute traumatic injury of the distal descending aorta associated with thoracic spine injury. Eur Radiol. 1998;8(1):60-2.

8 Magerl F, Aebi M, Gertzbein SD, Harms J, Nazarian S. A comprehensive classification of thoracic and lumbar injuries. Eur Spine J. 1994;3(4):184-201.

9 Divi SN, Schroeder GD, Oner FC, Kandziora F, Schnake KJ, Dvorak MF, et al. AOSpinespine trauma classification system: the value of modifiers: a narrative review with commentary on evolving descriptive principles. Global Spine J. 2019;9(1 Suppl):77S-88S.
10 Kepler CK, Vaccaro AR, Schroeder GD, Koerner JD, Vialle LR, Aarabi B, et al. The Thoracolumbar AOSpine Injury Score. Global Spine J. 2016;6(4):329-34.

11 Sixta S, Moore FO, Ditillo MF, Fox AD, Garcia AJ, Holena D, et al. Screening for thoracolumbar spinal injuries in blunt trauma: an Eastern Association for the Surgery of Trauma practice management guideline. J Trauma Acute Care Surg. 2012;73(5):S326-32.

12 Goldberg AL, Kershah SM. Advances in imaging of vertebral and spinal cord injury. J Spinal Cord Med. 2010;33(2):105-16.

13 Kopp R, Beisse R, Weidenhagen R, Piltz S, Hauck S, Becker CR, et al. Strategies for prevention and operative treatment of aortic lesions related to spinal interventions. Spine. 2007;32(25):E753-60.

14 Vesalius A, Bonetus T. Sect 2. Sepulchretum sive anatomia practica ex cadaveribus morbo denatis. Genevae; 1700. p. 290.

15 Domenicucci M, Ramieri A, Landi A, Melone AG, Raco A, Ruggiero M, et al. Blunt abdominal aortic disruption (BAAD) in shear fracture of the adult thoraco-lumbar spine: case report and literature review. Eur Spine J. 2011; 20(Suppl 2):S314-9.

16 Santoro G, Ramieri A, Chiarella V, Vigliotta M, Domenicucci M. Thoraco-lumbar fractures with blunt traumatic aortic injury in adult patients: correlations and management. Eur Spine J. 2018;27(S2):248-57.

$17 \mathrm{Yu}$ L, Baumann BM, Raja AS, Mower WR, Langdorf MI, Medak AJ, et al. Blunt traumatic aortic injury in the pan-scan era. Acad Emerg Med. 2020;27(4):291-6.
18 Inaba K, Kirkpatrick AW, Finkelstein J, Murphy J, Brenneman FD, Boulanger BR, et al. Blunt abdominal aortic trauma in association with thoracolumbar spine fractures. Injury. 2001;32(3):201-7.

19 Azizzadeh A, Keyhani K, Miller CC 3rd, Coogan SM, Safi HJ, Estrera AL. Blunt traumatic aortic injury: initial experience with endovascular repair. J Vasc Surg. 2009;49(6): 1403-8.

20 Lee WA, Matsumura JS, Mitchell RS, Farber MA, Greenberg RK, Azizzadeh A, et al. Endovascular repair of traumatic thoracic aortic injury: clinical practice guidelines of the Society for Vascular Surgery. J Vasc Surg. 2011;53(1): 187-92.

21 Heneghan RE, Aarabi S, Quiroga E, Gunn ML, Singh N, Starnes BW. Call for a new classification system and treatment strategy in blunt aortic injury. J Vasc Surg. 2016;64(1): 171-6.

22 Xenos ES, Abedi NN, Davenport DL, Minion DJ, Hamdallah O, Sorial EE, et al. Meta-analysis of endovascular vs open repair for traumatic descending thoracic aortic rupture. J Vasc Surg. 2008;48(5):1343-51.

23 Fox N, Schwartz D, Salazar JH, Haut ER, Dahm P, Black JH, et al. Evaluation and management of blunt traumatic aortic injury: a practice management guideline from the Eastern Association for the Surgery of Trauma. J Trauma Acute Care Surg. 2015;78(1): $136-46$. 